\section{Cache-Only Memory Architecture}

2 Josep TORRELlas

3 University of Illinois at Urbana-Champaign 4231 Siebel

4 Center, M/C-258, Urbana, IL, USA

\section{Synonyms \\ 6 COMA}

\section{Definition}

8 A Cache-Only Memory Architecture (COMA) is a 9 type of cache-coherent nonuniform memory access

10 (CC-NUMA) architecture. Unlike in a conventional 11 CC-NUMA architecture, in a COMA, every shared12 memory module in the machine is a cache, where each 13 memory line has a tag with the line's address and state. 14 As a processor references a line, it transparently brings 15 it to both its private cache(s) and its nearby portion of 16 the NUMA shared memory (Local Memory) - possibly 7 displacing a valid line from its local memory. Effectively, 18 each shared-memory module acts as a huge cache mem19 ory, giving the name COMA to the architecture. Since 20 the COMA hardware automatically replicates the data and migrates it to the memory module of the node that is currently accessing it, COMA increases the chances of data being available locally. This reduces the possibility of frequent long-latency memory accesses. Effectively, COMA dynamically adapts the shared data layout to the application's reference patterns.

\section{Discussion}

\section{Basic Concepts}

29 In a conventional CC-NUMA architecture, each node 30 contains one or more processors with private caches and 31 a memory module that is part of the NUMA shared 32 memory. A page allocated in the memory module of one node can be accessed by the processors of all other 33 nodes. The physical page number of the page speci- 34 fies the node where the page is allocated. Such node is 35 referred to as the Home Node of the page. The physi- 36 cal address of a memory line includes the physical page 37 number and the offset within that page. 38

In large machines, fetching a line from a remote 39 memory module can take several times longer than 40 fetching it from the local memory module. Conse- 41 quently, for an application to attain high performance, 42 the local memory module must satisfy a large fraction 43 of the cache misses. This requires a good placement of 44 the program pages across the different nodes. If the pro- 45 gram's memory access patterns are too complicated for 46 the software to understand, individual data structures 47 may not end up being placed in the memory module of 48 the node that access them the most. In addition, when a 49 page contains data structures that are read and written 50 by different processors, it is hard to attain a good page 51 placement.

In a COMA, the hardware can transparently elimi- 53 nate a certain class of remote memory accesses. COMA 54 does this by turning memory modules into large caches 55 called Attraction Memory (AM). When a processor 56 requests a line from a remote memory, the line is 57 inserted in both the processor's cache and the node's 58 AM. A line can be evicted from an AM if another line 59 needs the space. Ideally, with this support, the proces- 60 sor dynamically attracts its working set into its local 61 memory module. The lines the processor is not access- 62 ing overflow and are sent to other memories. Because a 63 large AM is more capable of containing a node's current 64 working set than a cache is, more of the cache misses are 65 satisfied locally within the node. 66

There are three issues that need to be addressed in 67 COMA, namely finding a line, replacing a line, and deal- 68 ing with the memory overhead. In the rest of this article, 69 these issues are described first, then different COMA 70 designs are outlined, and finally further readings are 71 suggested. 


\section{Finding a Memory Line}

74 In a COMA, the address of a memory line is a global 5 identifier, not an indicator of the line's physical location 6 in memory. Just like a normal cache, the AM keeps a tag 7 with the address and state of the memory line currently 78 stored in each memory location. On a cache miss, the 9 memory controller has to look up the tags in the local 80 AM to determine whether or not the access can be ser81 viced locally. If the line is not in the local AM, a remote 2 request is issued to locate the block.

83

COMA machines have a mechanism to locate a line 84 in the system so that the processor can find a valid copy 85 of the line when a miss occurs in the local AM. Differ6 ent mechanisms are used by different classes of COMA 87 machines.

One approach is to organize the machine hierarchically, with the processors at the leaves of the tree. Each 0 level in the hierarchy includes a directory-like structure, with information about the status of the lines present in the subtree extending from the leaves up to that level of the hierarchy. To find a line, the processing node issues a request that goes to successively higher levels of the tree, potentially going all the way to the root. The process stops at the level where the subtree contains the line. This design is called Hierarchical COMA $[2,7]$.

Another approach involves assigning a home node to each memory line, based on the line's physical 100 address. The line's home has the directory entry for the 101 line. Memory lines can freely migrate, but directory 102 entries do not. Consequently, to locate a memory line, 103 a processor interrogates the directory in the line's home 104 node. The directory always knows the state and location 105 of the line and can forward the request to the right node. 106 This design is called Flat COMA [12].

\section{Replacing a Memory Line}

108 The AM acts as a cache, and lines can be displaced 109 from it. When a line is displaced in a plain cache, it is 110 either overwritten (if it is unmodified) or written back 111 to its home memory module, which guarantees a place 112 for the line.

113 A memory line in COMA does not have a fixed 114 backup location where it can be written to if it gets 115 displaced from an AM. Moreover, even an unmodified 116 line can be the only copy of that memory line in the 117 system, and it must not be lost on an AM displace118 ment. Therefore, the system must keep track of the last copy of a line. As a result, when a modified or other- 119 wise unique line is displaced from an AM, it must be 120 relocated into another AM.

To guarantee that at least one copy of an unmodi- 122 fied line remains in the system, one of the line's copies is 123 denoted as the Master copy. All other shared copies can 124 be overwritten if displaced, but the master copy must 125 always be relocated to another AM. When a master copy 126 or a modified line is relocated, the problem is decid- 127 ing which node should take the line in its AM. If other 128 nodes already have one or more other shared copies of 129 the line, one of them becomes the master copy. Other- 130 wise, another node must accept the line. This process is 131 called Line Injection.

Different line injection algorithms are possible. One 133 approach is for the displacing node to send requests to 134 other nodes asking if they have space to host the line [7]. 135 Another approach is to force one node to accept the line. 136 This, however, may lead to another line displacement. 137 A proposed solution is to relocate the new line to the 138 node that supplied the line that caused the displacement 139 in the first place [8].

140

\section{Dealing with Memory Overhead}

141

A CC-NUMA machine can allocate all memory to 142 application or system pages. COMA, however, leaves a 143 portion of the memory unallocated to facilitate auto- 144 matic data replication and migration. This unallocated 145 space supports the replication of lines across AMs. 146 It also enhances line migration to the AMs of the ref- 147 erencing nodes because less line relocation traffic is 148 needed.

Without unallocated space, every time a line is 150 inserted in the AM, another line would have to be relo- 151 cated. The ratio between the allocated data size and the 152 total size of the AMs is called the Memory Pressure. If the 153 memory pressure is $80 \%$, then $20 \%$ of the AM space is 154 available for data replication. Both the relocation traffic 155 and the number of AM misses increase with the mem- 156 ory pressure [8]. For a given memory size, choosing an 157 appropriate memory pressure is a trade-off between the 158 effect on page faults, AM misses, and relocation traffic. 159 


\section{Flat COMA}

184 A design called Flat COMA makes it easy to locate a 185 memory line by assigning a home node to each memory 186 line [12] - based on the line's physical address. The line's 187 home has the directory entry for the line, like in a con188 ventional CC-NUMA architecture. The memory lines 189 can freely migrate, but the directory entries of the mem190 ory lines are fixed in their home nodes. At a miss on a 191 line in an AM, a request goes to the node that is keeping 192 the directory information about the line. The directory 193 redirects the request to another node if the home does 194 not have a copy of the line. In Flat COMA, unlike in a 195 conventional CC-NUMA architecture, the home node 196 may not have a copy of the line even though no pro197 cessor has written to the line. The line has simply been 198 displaced from the AM in the home node.

199 Because Flat COMA does not rely on a hierarchy to 200 find a block, it can use any high-speed network.

\section{Simple COMA}

202 A design called Simple COMA (S-COMA) [10] transfers 203 some of the complexity in the AM line displacement and relocation mechanisms to software. The general coher- 204 ence actions, however, are still maintained in hard- 205 ware for performance reasons. Specifically, in S-COMA, 206 the operating system sets aside space in the AM for 207 incoming memory blocks on a page- granularity basis. 208 The local Memory Management Unit (MMU) has map- 209 pings only for pages in the local node, not for remote 210 pages. When a node accesses for the first time a shared 211 page that is already in a remote node, the processor suf- 212 fers a page fault. The operating system then allocates a 213 page frame locally for the requested line. Thereafter, the 214 hardware continues with the request, including locating 215 a valid copy of the line and inserting it, in the cor- 216 rect state, in the newly allocated page in the local AM. 217 The rest of the page remains unused until future 218 requests to other lines of the page start filling it. Sub- 219 sequent accesses to the line get their mapping directly 220 from the MMU. There are no AM address tags to check 221 if the correct line is accessed. 222

Since the physical address used to identify a line in 223 the AM is set up independently by the MMU in each 224 node, two copies of the same line in different nodes are 225 likely to have different physical addresses. Shared data 226 needs a global identity so that different nodes can com- 227 municate. To this end, each node has a translation table 228 that converts local addresses to global identifiers and 229 vice versa.

\section{Multiplexed Simple COMA}

231

S-COMA sets aside memory space in page-sized 232 chunks, even if only one line of each page is present. 233 Consequently, S-COMA suffers from memory frag- 234 mentation. This can cause programs to have inflated 235 working sets that overflow the AM, inducing frequent 236 page replacements and resulting in high operating sys- 237 tem overhead and poor performance. 238

Multiplexed Simple COMA (MS-COMA) [1] elim- 239 inates this problem by allowing multiple virtual pages 240 in a given node to map to the same physical page at 241 the same time. This mapping is possible because all the 242 lines on a virtual page are not used at the same time. 243 A given physical page can now contain lines belonging 244 to different virtual pages if each line has a short vir- 245 tual page ID. If two lines belonging to different pages 246 have the same page offset, they displace each other 247 from the AM. The overall result is a compression of the 248 application's working set. 


\section{Further Readings}

251 There are several papers that discuss COMA and 252 related topics. Dahlgren and Torrellas present a more 253 in-depth survey of COMA machine issues [3]. There 254 are several designs that combine COMA and con255 ventional CC-NUMA architecture features, such as 256 NUMA with Remote Caches (NUMA-RC) [9], Reactive 257 NUMA [5], Excel-NUMA [14], the Sun Microsystems' 258 WildFire multiprocessor design [6], the IBM Prism 259 architecture [4], and the Illinois I-ACOMA architecture 260 [13]. A model for comparing the performance of COMA 261 and conventional CC-NUMA architectures is presented 262 by Zhang and Torrellas [15]. Soundarajan et al. [11] 263 describe the trade-offs related to data migration and 264 replication in CC-NUMA machines.

\section{Related Entries}

266 Cache-Coherent Non-Uniform Memory Access 267 (CC-NUMA) architecture

\section{Bibliography}

1. Basu S, Torrellas J (1998) Enhancing memory use in simple coma: multiplexed simple coma. In: International symposium on highperformance computer architecture, Las Vegas, February 1998

2. Burkhardt $\mathrm{H}$ et al (1992) Overview of the KSR1 computer system. Technical Report 9202001, Kendall Square Research, Waltham, February 1992

3. Dahlgren F, Torrellas J (1999) Cache-only memory architectures.
11. Soundararajan V, Heinrich M, Verghese B, Gharachorloo K, 299 Gupta A, Hennessy J (1998) Flexible use of memory for repli- 300 cation/migration in cache-coherent DSM multiprocessors. In: 301 International symposium on computer architecture, Barcelona, 302 June 1998

12. Stenstrom P, Joe T, Gupta A (1992) Comparative performance 304 evaluation of cache-coherent NUMA and COMA architectures. 305 In: International symposium on computer architecture, Gold 306 Coast, Australia, May 1992, pp 80-91 307

13. Torrellas J, Padua D (1996) The illinois aggressive coma multi- 308 processor project (I-ACOMA). In: Symposium on the frontiers 309 of massively parallel computing, Annapolis, October $1996 \quad 310$

14. Zhang Z, Cintra M, Torrellas J (1999) Excel-NUMA: toward 311 programmability, simplicity, and high performance. IEEE 312 Trans Comput 48(2):256-264. Special Issue on Cache Memory, 313 February 1999

15. Zhang Z, Torrellas J (1997) Reducing remote conflict misses: 315 NUMA with remote cache versus COMA. In: International 316 symposium on high-performance computer architecture, San 317 Antonio, February 1997, pp 272-281 IEEE Computer Magazine 32(6):72-79, June 1999

4. Ekanadham K, Lim B-H, Pattnaik P, Snir M (1998) PRISM: an integrated architecture for scalable shared memory. In: International symposium on high-performance computer architecture, Las Vegas, February 1998

5. Falsafi B, Wood D (1997) Reactive NUMA: a design for unifying S-COMA and CC-NUMA. In: International symposium on computer architecture, Denver, June 1997

6. Hagersten E, Koster M (1992) WildFire: a scalable path for SMPs. In: International symposium on high-performance computer architecture, Orlando, January 1999

7. Hagersten E, Landin A, Haridi S (1992) DDM - a cache-only memory architecture. IEEE Computer 25(9):44-54

8. Joe T, Hennessy J (1994) Evaluating the memory overhead required for COMA architectures. In: International symposium on computer architecture, Chicago, April 1994, pp 82-93

9. Moga A, Dubois M (1998) The effectiveness of SRAM network caches in clustered DSMs. In: International symposium on highperformance computer architecture, Las Vegas, February 1998

10. Saulsbury A, Wilkinson T, Carter J, Landin A (1995) An argument for simple COMA. In: International symposium on high-performance computer architecture, Raleigh, January 1995, pp 276-285 